\title{
Ring-Opening Polymerization of Sultones. VIII. Initial Reaction on Ring-Opening Polymerization of 3-Hydroxy-1-propane Sulfonic Acid Sultone with Tertiary Amines
}

\author{
Shizunobu Hashimoto and Takayuki Yamashita \\ Department of Applied Chemistry, Doshisha University, \\ Karasuma-imadegawa, Kamikyo-ku, Kyoto 606, Japan.
}

(Received March 14, 1975)

\begin{abstract}
The present paper describes the polymerization of 3-hydroxy-1-propane sulfonic acid sultone (PS) with tertiary amines at $80^{\circ} \mathrm{C}$.

The course of polymerization involved an early stage which was a slow initiation, a propagation step, and an equilibrium state between polymer and monomer. The slow initiation seemed to be the following reaction:
\end{abstract}

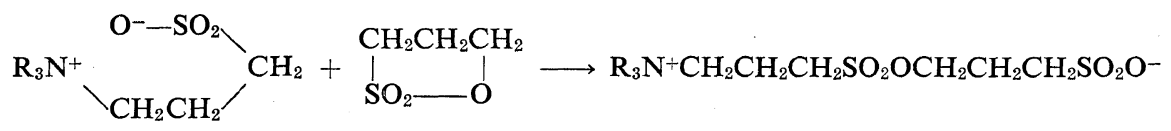

It was explained that the low reactivity of the anion side of the zwitterion is attributed to the intramolecular ion-pair. The low reactivity of the anion side of the zwitterion could be expected from the fact that the coulombic energy of charge separation in the intramolecular ion-pair is higher than that in the macrozwitterion.

This mechanism was explained practically from the rate of addition reaction of PS with tertiary amines in solvents, and from the formation of zwitterion in bulk polymerization, which was directly monitored by using an NMR spectrometer.

The rate constants $k_{\mathrm{p}}$ of propagation for quinoline, triethylamine, diethyl sulfide, and sulfobetain (formed from PS and diethylaniline) showed fairly close agreement with each other.

\section{KEY WORDS Ring-Opening Polymerization / Propane Sultone / Slow Initiation / Propagation / Sulfobetain / Zwitterion /}

In a previous paper we described the general features of the polymerization of cyclic sulfonic acid ester, 3-hydroxy-1-propane sulfonic acid sultone (PS) (I), with tertiary amines (II) or diethyl sulfide. ${ }^{1,2}$

The polymerization was shown to be initiated by zwitterion (sulfobetain) (III), which was formed by the addition reaction of PS to tertiary amines as an initiator (eq 1). Then an anionic living-polymerization through macrozwitterion (IV) occurred (eq 2): it proceeded by the reaction via alkyl-oxygen cleavage of the $O$-methylene group in the monomer. The equilibrium state between monomer (PS) and polymer was observed in this polymerization (eq 3 ).<smiles>[R16]NC1CCS(=O)(=O)O1</smiles>

(I)

$$
\mathrm{R}_{3} \mathrm{~N}^{+} \mathrm{CH}_{2} \mathrm{CH}_{2} \mathrm{CH}_{2} \mathrm{SO}_{2} \mathrm{O}^{-}
$$

$$
\begin{gathered}
(\mathrm{III})+n(\mathrm{I}) \stackrel{\longrightarrow}{\longrightarrow} \mathrm{R}_{3} \mathrm{~N}^{+}\left(\mathrm{CH}_{2} \mathrm{CH}_{2} \mathrm{CH}_{2} \mathrm{SO}_{2} \mathrm{O}\right)_{n^{-}} \\
\mathrm{CH}_{2} \mathrm{CH}_{2} \mathrm{CH}_{2} \mathrm{SO}_{2} \mathrm{O}^{-} \\
(\mathrm{IV}) \\
(\mathrm{IV})+(\mathrm{I}) \stackrel{{ }^{k} \mathrm{p}}{\stackrel{k_{-p}}{\longrightarrow}} \mathrm{R}_{3} \mathrm{~N}^{+}\left(\mathrm{CH}_{2} \mathrm{CH}_{2} \mathrm{CH}_{2} \mathrm{SO}_{2} \mathrm{O}\right)_{n+1^{-}} \\
\mathrm{CH}_{2} \mathrm{CH}_{2} \mathrm{CH}_{2} \mathrm{SO}_{2} \mathrm{O}^{-}
\end{gathered}
$$

In this polymerization, if the zwitterion (III) is formed rapidly in good yield as shown in eq 1 , the rate of polymerization is given by following equation.

$$
\frac{-\mathrm{d}[\mathrm{M}]}{\mathrm{d} t}=k_{\mathrm{p}}\left[\mathrm{P}^{*}\right][\mathrm{M}]-k_{-\mathrm{p}}\left[\mathrm{P}^{*}\right]
$$

In eq $4,[M]$ is the concentration of monomer, 


\section{S. Hashimoto and T. Yamashita}

$\left[\mathrm{P}^{*}\right]$ is the concentration of growing chains and is equal to the concentration of initiator, $k_{\mathrm{p}}$ is the rate constant of propagation, and $k_{-p}$ is the rate constant of depolymerization.

Integration of eq 4 , with $[\mathrm{M}]=[\mathrm{M}]_{0}$ when $t=0$, gives eq 5 .

$$
k_{\mathrm{p}} t=\frac{1}{\left[\mathrm{P}^{*}\right]} \ln \frac{[\mathrm{M}]_{0}-[\mathrm{M}]_{\mathrm{e}}}{[\mathrm{M}]-[\mathrm{M}]_{\mathrm{e}}}
$$

Here, $[M]_{e}$ is the monomer concentration in the equilibrium state and $[\mathrm{M}]_{0}$ is the initial monomer concentration.

In PS polymerization, an S-shaped curve was obtained for the time-conversion curves. As shown in Figure 1, except for the early stage, the plot of eq 5 shows a fairly linear fit.

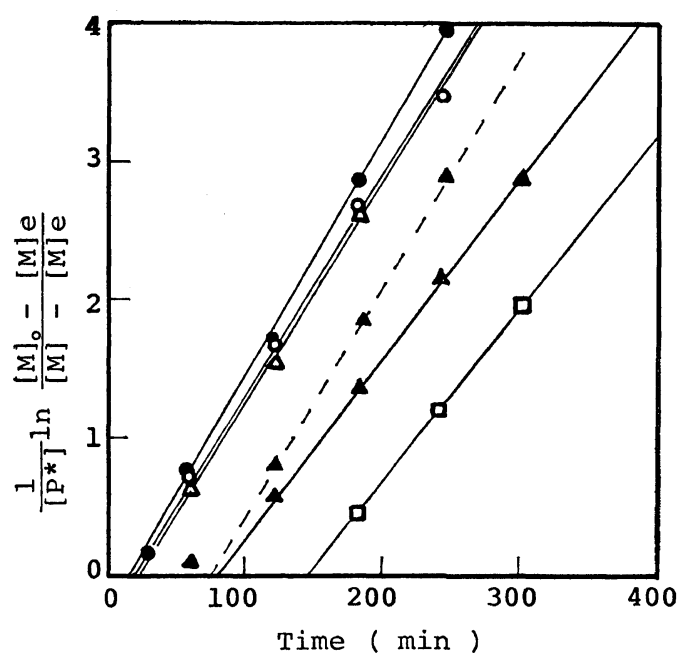

Figure 1. Time $-\frac{1}{\left[\mathrm{P}^{*}\right]} \ln \frac{[\mathrm{M}]_{0}-[\mathrm{M}]_{\mathrm{e}}}{[\mathrm{M}]-[\mathrm{M}]_{\mathrm{e}}}$ curves for the bulk polymerization of PS. Solid line: $\bullet$, quinoline $(0.45 \mathrm{~mol} / l) ; \triangle$, diethyl sulfide $(0.46 \mathrm{~mol} / l) ; \square$, pyridine $(0.48 \mathrm{~mol} / l)$; $A$, diethylaniline $(0.42 \mathrm{~mol} / l)$; $\bigcirc$, triethylamine $(0.46 \mathrm{~mol} / l)$. Broken line: $\mathbf{A}$, sulfobetain $(0.45 \mathrm{~mol} / l)$ formed by the addition of PS and diethylaniline; $80^{\circ} \mathrm{C}$.
From the slope of this straight line, the rate constant $k_{\mathrm{p}}$ of the propagation can be evaluated. The rate constant $k_{\mathrm{p}}$ and the induction period (early stage) are shown in Table I.

The $k_{\mathrm{p}}$ for quinoline, triethylamine, and diethyl sulfide show a close agreement with each other, but $k_{\mathrm{p}}$ for pyridine and diethylaniline are much lower than those of the others. From the close agreement of $k_{\mathrm{p}}$ for quinoline, triethylamine, and diethyl sulfide, the relation of $\left[\mathrm{P}^{*}\right]=$ [initiator] in eq 5 has been found to hold.

On the other hand, the induction period decreases in the following order: pyridine $\gg$ diethylaniline $\gg$ diethyl sulfide $>$ triethylamine $>$ quinoline. This is in conflict with the rate of eq 1 , which was expected from the basicity of the initiator.

To explain the slow initiation, more details of the initial propagation step were studied in the following manner: First, the rate of equimolar addition reaction of PS and tertiary amines was determined in benzene and acetonitrile. Secondly, the formation of zwitterion from initiator and PS in bulk polymerization of PS was directly monitored by using an NMR spectrometer.

\section{RESULTS AND DISCUSSION}

Addition Reaction of PS and Tertiary Amines

The reaction of sultone with tertiary amines to form the sulfobetain (zwitterion, III) is well known. ${ }^{3}$

The addition reaction was carried out in acetonitrile or benzene at reflux. The timeconversion curves of PS to III are plotted in Figures 2 and 3.

If this addition reaction proceeds via $S_{\mathrm{N}} 2$ reaction, the rate of addition is given by eq 6 .

$$
\frac{-\mathrm{d}[\mathrm{PS}]}{\mathrm{d} t}=k_{\mathrm{i}}[\mathrm{PS}][\mathrm{TA}]
$$

Table I. Rate constant $k_{\mathrm{p}}$ and induction period of bulk polymerization of PS with tertiary amines or diethyl sulfide $\left(80^{\circ} \mathrm{C}\right)$

\begin{tabular}{cccccc}
\hline \multicolumn{1}{c}{ Initiator } & Quinoline & Triethylamine & Diethylaniline & Pyridine & Diethyl sulfide \\
\hline$k_{\mathrm{p}} \times 10^{-4}, 1 / \mathrm{mol} \mathrm{sec}$ & 2.72 & 2.75 & $2.03(2.76)^{\mathrm{a}}$ & 1.98 & 2.72 \\
Induction period, min & 16 & 20 & 65 & 140 & 30 \\
\hline
\end{tabular}

a Rate constant $k_{\mathrm{p}}$ of PS bulk polymerization with sulfobetain formed by the addition reaction of PS and diethylaniline. 
Here, [PS] is the monomer concentration, [TA] is the concentration of tertiary amines, and $k$ is the rate constant of the addition reaction.

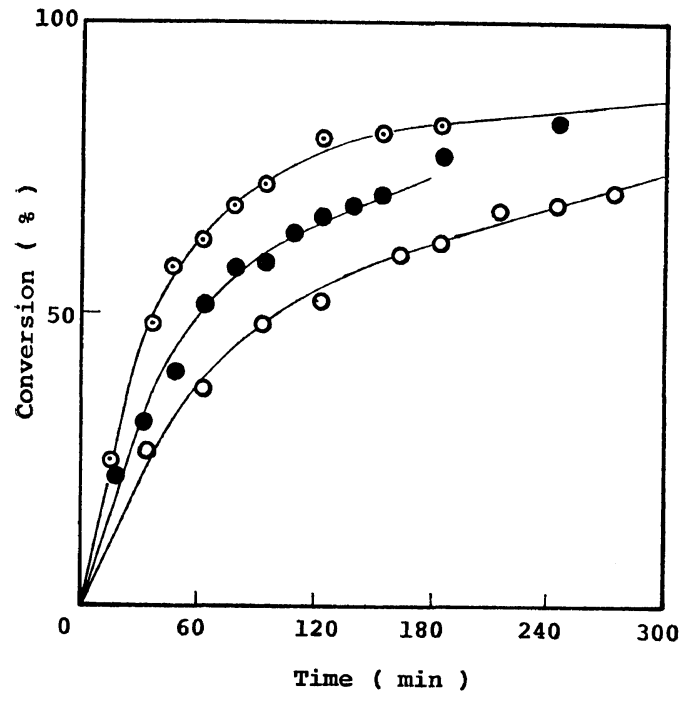

Figure 2. Addition reaction of various tertiary amines to PS in acetonitrile at $82^{\circ} \mathrm{C}$ (reflux): $\mathrm{O}$, quinoline $1.0 \mathrm{~mol} / l$, PS $1.0 \mathrm{~mol} / l$; $\odot$, triethylamine $0.5 \mathrm{~mol} / l$, PS $0.5 \mathrm{~mol} / l$; 0 , pyridine $0.2 \mathrm{~mol} / l$, PS $0.2 \mathrm{~mol} / \mathrm{l}$.

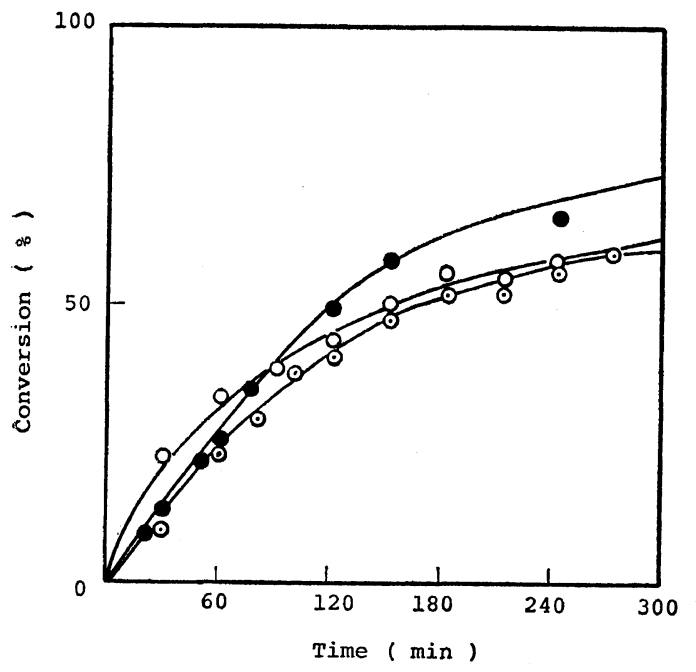

Figure 3. Addition reaction of various tertiary amines to PS in benzene at $80^{\circ} \mathrm{C}$ (reflux): $\bigcirc$, quinoline $1.0 \mathrm{~mol} / l$, PS $1.0 \mathrm{~mol} / l$, $\odot$, triethylamine $0.5 \mathrm{~mol} / l$, PS $0.5 \mathrm{~mol} / l$; $\bigcirc$, pyridine $0.5 \mathrm{~mol} / l$, PS $0.5 \mathrm{~mol} / l$.
Integration of eq 6 with $[\mathrm{PS}]=[\mathrm{TA}]$ and $[\mathrm{PS}]=$ $[\mathrm{PS}]_{0}$ at $t=0$ gives eq 7 .

$$
k_{\mathrm{i}} t=\frac{[\mathrm{PS}]_{0}-[\mathrm{PS}]}{[\mathrm{PS}][\mathrm{PS}]_{0}}
$$

Here, $[\mathrm{PS}]_{0}$ is the initial concentration of PS. Figure 4 shows that the data in Figures 2 and 3 give a linear relationship of $\left([\mathrm{PS}]_{0}-[\mathrm{PS}]\right) /[\mathrm{PS}][\mathrm{PS}]_{0}$ against the reaction time, as expected from eq 7 .

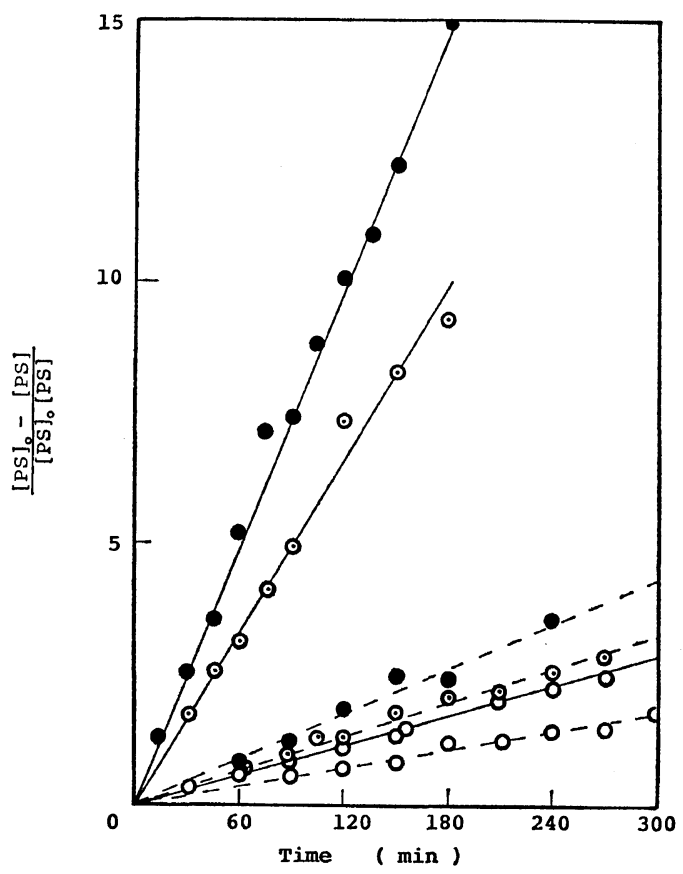

Figure 4. Application of the experimental data of the addition reaction of PS and tertiary amines to eq 7. Solid line, in acetonitrile and broken line, in benzene: $\bigcirc$, quinoline; $\odot$, triethylamine; , pyridine.

Table II. Rate constants $k_{\mathrm{i}}$ of addition reaction of various tertiary amines and PS in solvent at reflux

\begin{tabular}{lccc}
\hline \multirow{2}{*}{ Amine } & \multicolumn{2}{c}{$k_{\mathrm{i}} \times 10^{-4}, 1 / \mathrm{mol} \mathrm{sec}$} \\
\cline { 2 - 3 } & Acetonitrile & Benzene & \\
& Act/mol sec & \\
\hline Quinoline & 1.53 & 0.92 & \\
Triethylamine & 8.91 & 1.72 & $1.13 \times 10^{-6}$ \\
Pyridine & 13.3 & 2.46 & $1.55 \times 10^{-6}$ \\
\hline
\end{tabular}

a Rate constant of Menschutkin reaction of isopropyl iodide and tertiary amines in nitrobenzene at $25^{\circ} \mathrm{C}^{4}$. 
The rate constant $k_{\mathrm{i}}$ was evaluated from the slope in Figure 4 and is shown in Table II.

The order for $k_{\mathrm{i}}$ in the addition reaction of pyridine and triethylamine with PS agreed well with that in the Menschutkin reaction of isopropyl iodide and tertiary amines in nitrobenzene at $25^{\circ} \mathrm{C}$. The formation of sulfobetain from PS and diethylaniline was much slower than that of the other amines.

\section{Bulk Polymerization of PS}

The $k_{\mathrm{i}}$ in Table II are in conflict with the order for the induction period. This fact indicates that the slow initiation is not dependent on the rate of eq 1 .

To obtain some clarification of the slow initiation mechanism, the polymerization of PS with sulfobetain was examined, and the result is plotted in Figure 1 using eq 5 .

The induction period was the same as that of the polymerization with diethylaniline, and $k_{\mathrm{p}}$ was greater than that of the polymerization with diethylaniline.

The $k_{p}$ for sulfobetain was also in fair agreement with $k_{\mathrm{p}}$ for quinoline, triethylamine, and diethyl sulfide.

Consequently, this suggests that the early stage was independent of the rate of eq 1 , and $\left[\mathrm{P}^{*}\right]$ in the PS polymerization with diethylaniline was smaller than the concentration of initiator.

In the bulk polymerization of PS with quinoline and pyridine at $80^{\circ} \mathrm{C}$, the formation of zwitterion (sulfobetain) from initiator and monomer was directly monitored by using an NMR spectrometer.

Figure 5 shows the NMR spectrum of the bulk polymerization of PS.

The NMR spectra (Figure 5a) at 10 min after the beginning of polymerization consist of peaks caused by the monomer, the quinolinium cation group, and a very small amount of quinoline remaining in the system.

After $30 \mathrm{~min}$, the NMR peaks due to the methylene adjacent to the quinolinium cation appeared (Figure 5b, $\equiv \mathrm{N}^{+}-\mathrm{CH}_{2}-; 5.3 \mathrm{ppm}$ ).

In the polymerization with pyridine, the NMR spectra consisted of peaks due to the pyridinium cation group ( $8-9 \mathrm{ppm})$, the methylene $(4.9 \mathrm{ppm})$ adjacent to the pyridinium cation group, and the monomer at $10 \mathrm{~min}$ after the beginning of the PS polymerization (Figure 6).

These NMR peaks were identified by comparing this NMR spectrum with that of sulfobetain and polymer. ${ }^{1}$

From these experimental facts, it seems to be quite all right to consider that the reaction of

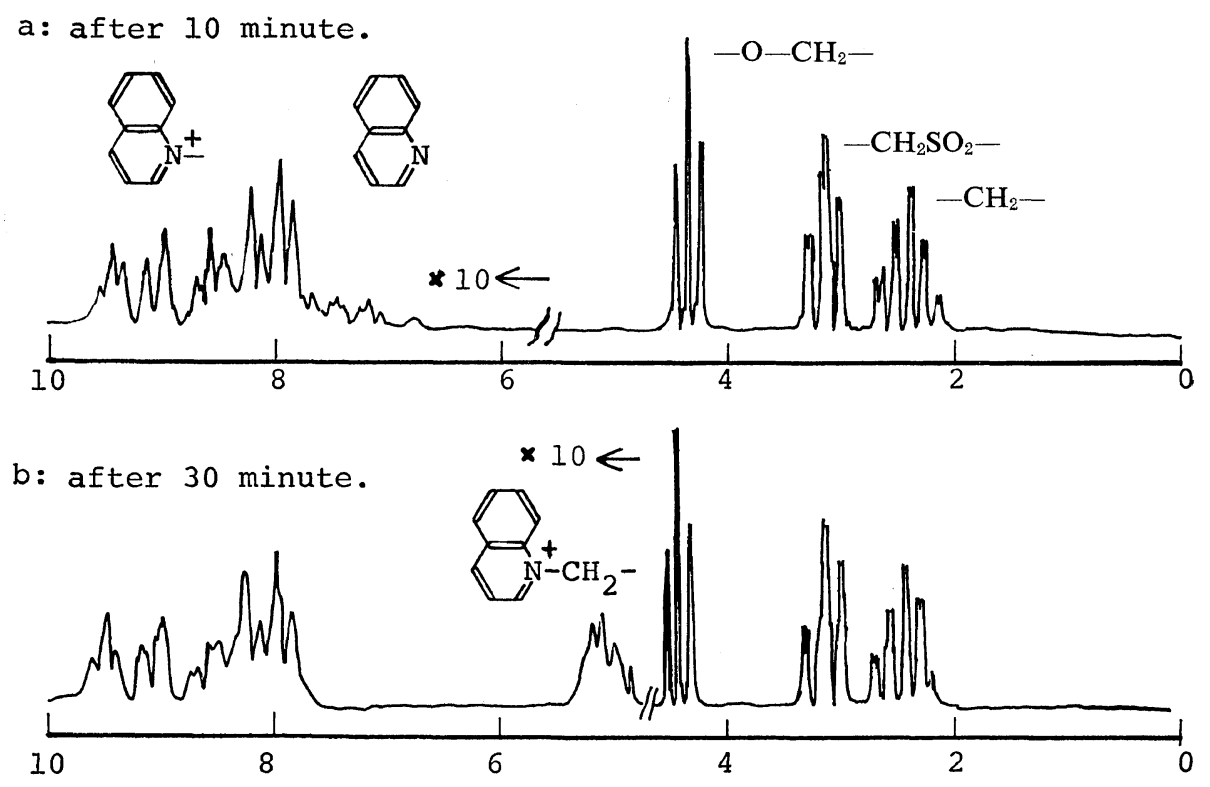

Figure 5. Bulk polymerization of PS in NMR sample tube: PS, $11.09 \mathrm{~mol} / l$; quinoline, $0.47 \mathrm{~mol} / l$; $80^{\circ} \mathrm{C}$. 


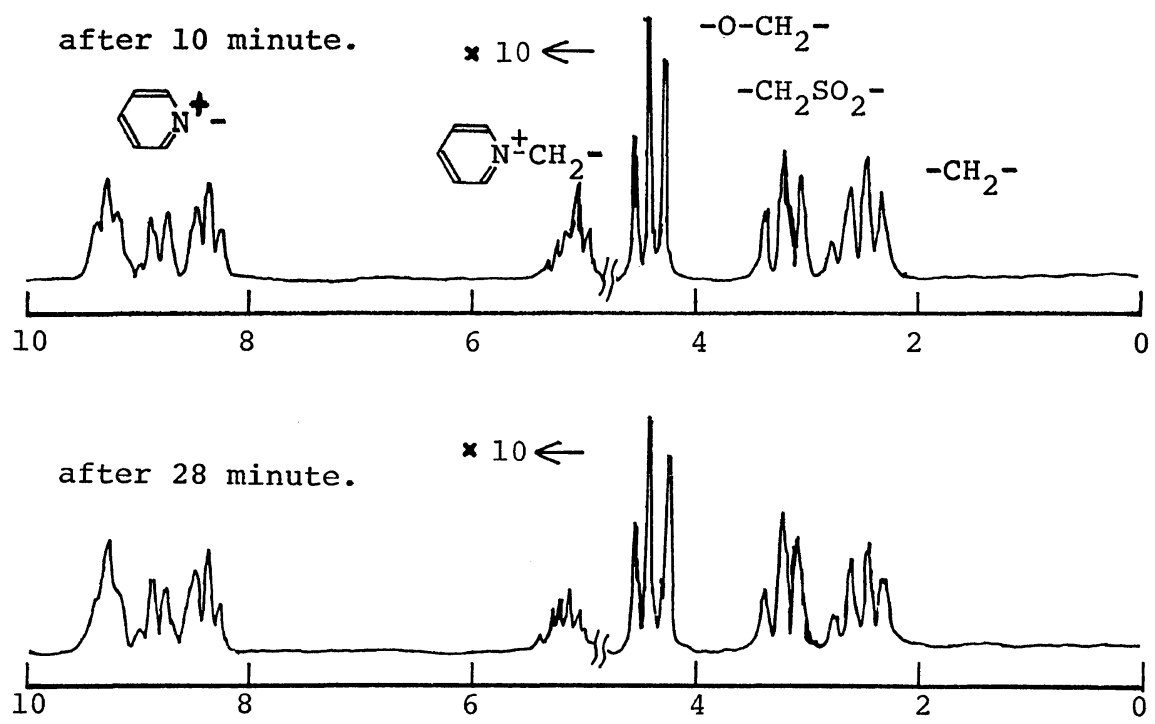

Figure 6. Bulk polymerization of PS in NMR sample tube: PS, $11.09 \mathrm{~mol} / \mathrm{l}$; pyridine, $0.79 \mathrm{~mol} / \mathrm{l}$; $80^{\circ} \mathrm{C}$.

eq 1 proceeds rapidly to form the zwitterion in good yield in the presence of a large excess of monomer without solvent.

Accordingly, assuming that the reaction of eq 1 proceeds rapidly, and $k_{\mathrm{p}}$ for propagation with each initiator are in fair agreement with each other, the rate-determining step at the slow initiation seems to be the following reaction (eq 8)

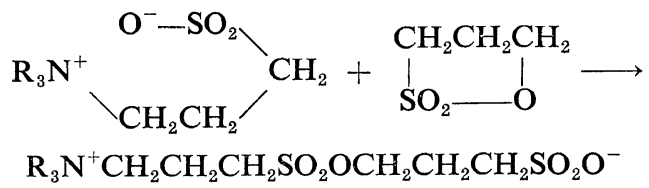

This conclusion would be explained in the following manner: The low reactivity of the anion side of the zwitterion can be expected from the effect of the neighboring quarternary ammonium cation, and the coulombic energy of charge separation in eq 8 would be higher than that in the propagation step (eq 9)

$$
\begin{aligned}
& m \mathrm{CH}_{2} \mathrm{SO}_{2} \mathrm{O}^{-}+\underset{\mathrm{SO}_{2}-\mathrm{O}_{2}}{\mathrm{CH}_{2} \mathrm{CH}_{2} \mathrm{CH}_{2}} \stackrel{k_{\mathrm{p}}}{\longrightarrow} \\
& m \mathrm{CH}_{2} \mathrm{SO}_{2} \mathrm{OCH}_{2} \mathrm{CH}_{2} \mathrm{CH}_{2} \mathrm{SO}_{2} \mathrm{O}^{-}
\end{aligned}
$$

In such a case, the zwitterion (III) cyclizes to form an intramolecular ion-pair.

From the above description, it was concluded that the polymerization of PS with tertiary amines proceeded via eq 1,8 , and 9 , and the induction period was observed for eq 8 . This was explained by the reactivity of the anion side of zwitterion. The reactivity of zwitterion is affected by the electron-attractive effect of the quarternary ammonium cation on the intramolecular ion-pair.

After eq 8 was completed, polymerization proceeded without termination. The propagation step was not affected by the initiator, and it proceeds by the reaction of the sulfonate anion with polymer and monomer (eq 9).

Consequently, the propagation rate constants $k_{\mathrm{p}}$ for quinoline, triethylamine, diethyl sulfide, and sulfobetain (formed from PS and diethylaniline) showed fairly close agreement with each other.

For the ring-opening polymerization of $\beta$ propiolactone and 2-methyl-2-oxazoline, it has been reported that the early step of polymerization was affected by the reactivity of zwitterion formed from monomer and initiator. ${ }^{5,6}$

\section{EXPERIMENTAL}

\section{Reagents}

The monomer PS was a commercial sample. It was dried with sodium sulfate and purified by repeated distillation under nitrogen; bp 129.5- 


\section{S. Hashimoto and T. Yamashita}

$131^{\circ} \mathrm{C} / 8.5-9 \mathrm{mmHg}$. The quinoline, pyridine, diethyl sulfide, triethylamine, and diethylaniline were commercial samples, and were distilled under nitrogen before use. Acetonitrile and benzene were dried with $\mathrm{P}_{2} \mathrm{O}_{5}$ or metallic sodium and distilled.

\section{Addition Reaction of PS and Tertiary Amines}

The rates of reaction were measured by gaschromatography using an internal standard. The reaction was carried out at the equimolar of PS and tertiary amines at reflux in benzene or acetonitrile. The amounts of PS were measured at appropriate intervals of time, and rate constants were evaluated from the plots of Figure 4.

Chromatograph:

Column: Silicon SE-30 (5\%) $2 \mathrm{~m}$

Carrier gas: $\mathrm{H}_{2}(60 \mathrm{~m} l / \mathrm{min})$

Bulk Polymerization of PS with Tertiary Amines or Diethyl Sulfide

Polymerization was carried out in a sealed tube. PS was placed into a glass tube and was dried under high vacuum. Then the glass tube was filled up with dry nitrogen gas. An initiator was added to this solution with a microsyringe. After the glass tube was sealed, it was left in the thermostat bath for the required time.

After the polymerization, the reaction mixture was poured into excess methanol, and the precipitated polymer was separated by filtration, washed with methanol, and weighed.
The progress of change of PS and initiator to zwitterion in the early step of PS polymerization was directly determined by means of NMR spectroscopy. PS was introduced into an NMR sample tube at $80^{\circ} \mathrm{C}$, and then tertiary amine as an initiator was added. The polymerization system was monitored by recording NMR spectra at several reaction times on a Varian HA-100 NMR spectrometer.

Bulk Polymerization of PS with Sulfobetain

Equimolar amounts of PS and diethylaniline were charged into a glass tube. The glass tube was placed in the thermostat bath $\left(80^{\circ} \mathrm{C}\right)$ for $4 \mathrm{hr}$. And then PS as a monomer was introduced in this glass tube.

Polymerization was carried out in the same manner as described above.

\section{REFERENCES}

1. S. Hashimoto, T. Yamashita, and M. Kaneda, Kobunshi Kagaku (Chem. High Polymers), 30, 244 (1973).

2. S. Hashimoto, T. Yamashita, and M. Kaneda, Polymer J., 6, 238 (1974).

3. J. H. Helberger, Ann., 588, 71 (1954).

4. H. C. Brown and N. R. Eldred, J. Amer. Chem. Soc., 71, 445 (1949).

5. T. Saegusa, H. Ikeda, and H. Fujii, Macromolecules, 5, 359 (1971).

6. N. Mathes and V. Jaacks, Makromol. Chem., 142, 209 (1971). 